\title{
Relação entre força muscular e área de secção transversa muscular em adultos jovens sedentários
}

Ronei Silveira Pinto, Gianni Rodolfi e Lucimere Bohn

\begin{abstract}
Resumo
A proposta deste estudo foi determinar a relação entre a área de secção transversa muscular ASTm) estimada e a força máxima dinâmica de extensores de joelho e flexores do cotovelo. A amostra foi constituída de adultos jovens do sexo masculino, sedentários e aparentemente saudáveis $(\mathrm{n}=10)$. A força máxima dinâmica foi avaliada através do teste de uma repetição máxima (1-RM). As estimativas da ASTm de coxa e braço foram calculadas a partir da equação de Gurney (1973). Os resultados não mostraram correlação entre as variáveis força máxima dinâmica e a estimativa da área de secção transversa muscular $(\mathrm{p}>0,05)$ e uma grande varinabilidade amostrai. Essa pode ter sido in-fluenciada por fatores como o nível de atividade física (que influenciaria na coordenação neuromuscular e nos mecanismos inibitórios) e pela predominância dos diferentes tipos de fibras musculares em cada sujeito. Estes resultes impossibilitam a modulação da carga de treinamento a partir da ASTm, tendo em vista a rrande variabilidade apresentada.
\end{abstract}

U nitermos: Força muscular, antropometría, área de secção transversa muscular

\section{Abstract}

* purpose of this study was to determine the

- $\quad$ : hip between muscle cross-sectional área

-zmãted and máximum dynamic strength ofknee xtension and elbow flexión. The sample was wistituded by healthy and untrained young ma- les $(n=10)$. The máximum dynamic strength was measured by the test one máximum repetition ( $T$ RM). The estimate of muscle cross-sectional área was calculate with the Gurney equation (1973). The results do not showed relationship between máximum dynamic strength and muscle crosssectional área estimated $(p>0,05)$ and a great variability ofthe subjeets. This variability can be influenced by factor with the levei of physical activity (affecting the neuromuscular coordenation and the inhibitory mechanisms) and by variation in the distribution of dijferent fibre types in each subjects. These results make impossible the modulation of the load of training through muscle cross-sectional área Um dos pontos estimated, due to the great fundamentais na variability founded in the elaboração de um
available subjects. programa de

\section{Introdução} treinamento de força é o treinamento de força é de treino. Essa é uma das atividades físicas normalmente mais difundidas atualmente, realizada a partir $d a$ sendo que nos Estadosexperiência do Unidos é o tipo de professor I instrutor, treinamento físico individual por tentativas e erros. mais popular (Baechle,

1998). Entre os praticantes estão pessoas de idades variadas, desde jovens até idosos. Esta prática tem diversos objetivos, sendo realizada para fins profiláticos, estéticos, esportivos e terapêuticos, em clubes, clínicas de recuperação, academias, etc. 
Um dos pontos fundamentais na elaboração de um programa de treinamento de força é a modulação da carga de treino. Essa é normalmente realizada a partir da experiência do professor / instrutor, por tentativas e erros. Beachle (1998) propõe que a modulação da carga de treino seja realizada tendo-se como referência o peso corporal, que é multiplicado por coeficientes $O$ conhecimento ${ }_{\text {específicos, de acordo }}^{\text {es }}$ da área muscular específicos. Entretanto, a envolvida no $\begin{aligned} & \text { dependência da força } \\ & \text { de }\end{aligned}$ movimento específico sobre o peso corporal poderia ser útil na somente é vista de forma determinação da clara em indivíduos com intensidade do as mesmas qualificações treinamento, uma físicas. Para aqueles que vez que cada atividades físicas ou centímetro quadrado desportivas, a correlação $\left(\mathrm{cm}^{2}\right)$ de área entre o peso corporal e a muscular pode força máxima tem sido produzir uma força considerada baixa, determinada $\mid \begin{aligned} & \text { podendo ser igual a zero } \\ & \text { (Zatziorskcy, } 1999) .\end{aligned}$

Outra forma de se modular a carga de treino é através do teste de uma repetição máxima (1$\mathrm{RM})$, com o qual se determina a intensidade (carga) de cada exercício utilizando-se percentuais do valor avaliado (Badillo, 1997; Fleck e Kraemer, 1999; Mayhew et al., 1993). Este método é eficaz e aceito pela comunidade científica internacional, sendo utilizado em vários estudos relacionados a este tema (Badillo, 1997; Baechle, 1998; Fleck e Kraemer, 1999).

A força máxima dinâmica, expressa pelo teste de 1-RM, é determinada, principalmente, por dois fatores: a coordenação neuromuscular e a área de secção transversa muscular (ASTm) envolvida no movimento. Outros fatores podem também influenciar na determinação desse valor, dentre os quais os fatores hormonais (a síntese de testosterona e do hormônio do crescimento) e a arquitetura muscular envolvidos no movimento (ângulo de penação das fibras musculares) (Lehmkuhl e Smith, 1989; Kraemer, 1992; Mayhew et al., 1991).

A coordenação neuromuscular pode ser avaliada através da estimulação elétrica induzida, bem como pela eletromiografia integrada (IEMG) (Badillo, 1997; Sale, 1992). Estes métodos, apesar de tecnicamente adequados, são restritos a Laboratórios de Pesquisas e de pouca aplicabilidade.

De outro lado, a ASTm é igualmente avaliada através de técnicas laboratoriais sofisticadas, dentre as quais estão a ressonância magnética e a tomografia computadorizada (Gurneey e Jelliffe, 1973; Heymesfield et al., 1982;Maughan, 1983;Rolland-Cachera, 1997), também pouco aplicáveis.

No entanto, Cureton (1988), através de um estudo realizado com homens e mulheres submetidos a um treinamento de força de 16 semanas, demonstrou uma boa relação entre a ASTm estimada a partir de dobras cutâneas e perímetros musculares e a ASTm avaliada por tomografia computadorizada. Segundo Mayhew (1991), este simples procedimento antropométrico pode ser adequadamente utilizado para a estimativa da ASTm, além de apresentar boa aplicabilidade na área do treinamento físico. $\mathrm{O}$ conhecimento da área muscular envolvida no movimento específico poderia ser útil na determinação da intensidade do treinamento, uma vez que cada centímetro quadrado $\left(\mathrm{cm}^{2}\right)$ de área muscular pode produzir uma força determinada (Lehmkuhl e Smith, 1989).

Sendo assim, o objetivo deste estudo foi correlacionar as estimativas de ASTm dos pontos médios do braço e da coxa com a força dinâmica máxima avaliada através dos testes de 1-RM de flexão do cotovelo e extensão do joelho, respectivamente. 


\section{Material e Métodos}

Foram selecionados, intencionalmente, 10 indivíduos homens, adultos jovens, sedentários e aparentemente saudáveis, que, há um ano, não realizavam nenhuma atividade física sistemática e orientada.

\section{Avaliação Antropométrica}

Para caracterização da amostra, foram realizadas as mediadas de peso e estatura. Além disso, avaliou-se dobras cutâneas (tríceps e coxa) e perimetria (braço e coxa) segundo descrição de Lohman (1988), utilizando-se como resultado o valor intermediário obtido a partir de três medidas. Os procedimentos antropométricos foram realizados pelo mesmo avaliador e refeitos após uma semana, observando, desse modo, a fidedignidade das medidas.

O equipamento utilizado foi o compasso de dobras cutâneas da marca Lange $(1 \mathrm{~mm})$ e uma fita métrica flexível Lufkin $(1 \mathrm{~mm})$. Para mensuração da altura e do peso foi utilizada uma balança e um estadiômetro da marca Filizola

resolução de $100 \mathrm{~g}$ para peso e $0,5 \mathrm{~cm}$ para altura).

\section{Estimativas de Área de Secção Transversa Muscular (ASTm)}

As equações utilizadas para estimar a ASTm de coxa e braço, a partir de dobra cutânea e perímetro, são as propostas por Gurney (1973).

ASTm $=(\text { Per. }-\mathbf{T C x} \text { Dobra Cut. })^{2} / 4$ TI

Per. $=$ Perímetro de braço ou coxa.

Dobra Cut. $=$ Dobra cutânea de tríceps ou coxa.

\section{Avaliação da Força Máxima Dinâmica (1-RM)}

Utilizou-se o teste de uma repetição máxima (1RM), descrito por Knuttgen e Kraemer (1987), no qual se avaliou a máxima carga deslocada em uma única execução e em toda a amplitude dos movimentos de flexão do cotovelo e extensão do joelho em cinco segundos.

Os exercícios de flexão do cotovelo e extensão do joelho foram realizados de forma unilateral, apenas com os segmentos do lado direito, devido ao déficit bilateral (Flecke Kraemer, 1999; Sale, 1992).

\begin{tabular}{|l|l|l|}
\hline $\mathrm{N}=10$ & Média & D. P. \\
\hline Idade (anos) & 23,4 & 3,89 \\
Estatura (cm) & 176,35 & 7,45 \\
Peso Corporal (kg) & 70,98 & 6,21 \\
Perímetro do Braço (cm) & 28,95 & 1,53 \\
Perímetro de Coxa (cm) & 49,42 & 2,26 \\
Dobra de Tríceps (mm) & 10,9 & 4,28 \\
Dobra de Coxa (mm) & 12,5 & 4,30 \\
ASTm do Braço (cm²) & 52,02 & 6,31 \\
ÁST m da Coxa (cm²) & 165,20 & 19,03 \\
1-RM de Flexão de Cotovelo (kg) & 10,3 & 1,74 \\
1-RM de Extensão de Joelho (kg) & 30,8 & 4,09 \\
\hline
\end{tabular}

Tabela 1. Características Físicas, Antropométricas e de Força Máxima Dinâmica da Amostra

O equipamento utilizado para o teste de extensão do joelho foi da marca Taurus, modelo 
Extensión, sendo a resistencia caracterizada através de polias de raio simétrico.

O aparelho utilizado para realização do teste de flexão do cotovelo foi o de polia baixa da marca Taurus, modelo Flexión, que também apresenta polias de raio simétrico. A resolução das cargas foi de 250 gramas.

\section{Análise Estatística}

Para se estabelecer o perfil do grupo avaliado, com média e desvio padrão, foi utilizada a estatística descritiva.

Para se testar a fidedignidade das medidas antropométricas entre teste e reteste, foi utilizado o teste de correlação de Pearson, com índice de significancia aceito de 0,05 .

Para se correlacionar as variáveis força máxima e a ASTm foi utilizado o teste de correlação de Pearson, com índice de significancia aceito de 0,05 .

O pacote estatístico utilizado foi SPSS Versão 8.0.

\section{Resultados}

As características físicas e as médias dos valores dos testes de força máxima dinâmica (1-RM) de flexão do cotovelo e extensão do joelho são apresentados na Tabela 1 .

A fidedignidade das medidas de perímetros e dobras cutâneas foi avaliada e a correlação entre teste e reteste foi de $0,97, \operatorname{com} p<0,05$.

Não foi encontrada correlação entre as estimativas de ASTm do ponto médio da coxa calculada pela equação de Gurney (1973) e a força máxima dinâmica (1-RM) de extensão do joelho $(r=$ $-0,015 ; \mathrm{p} » 0,05)$. A figura 1 apresenta a dispersão da amostra.

Não foi encontrada correlação entre a estimativa de
ASTm do ponto médio do braço, calculada a partir da equação de Gurney (1973), e a força máxima dinâmica (1-RM) de flexão do cotovelo $(r=0,5 ; p>$ $0,05)$. A figura 2 apresenta a dispersão da amostra.

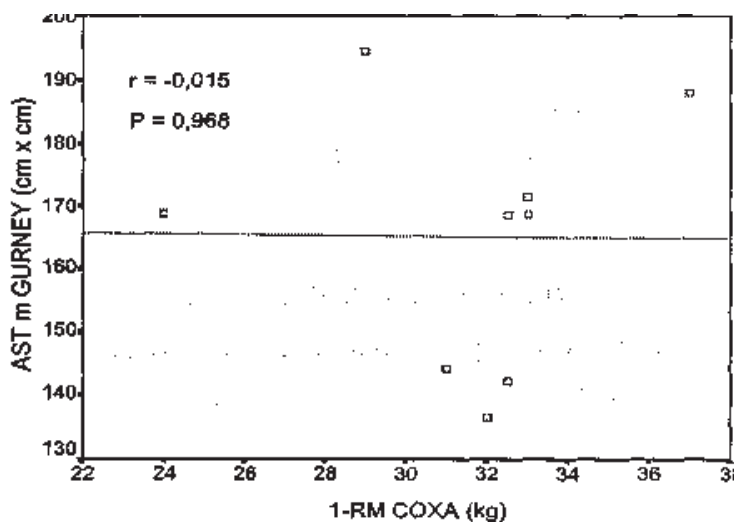

Figura 1. Correlação entre 1-RM de extensão de joelho e ASTm da coxa.

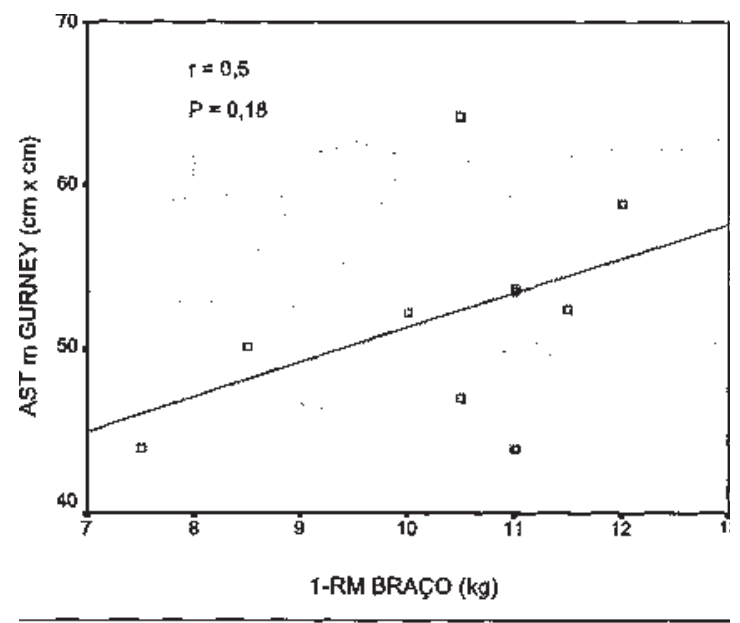

Figura 2. Correlação entre 1-RM de flexão do cotovelo e ASTm do braço.

\section{Discussão}

Alguns estudos apontam que a máxima força voluntária de extensores do joelho e flexores do cotovelo são positivamente correlacionadas com a ASTm de homens adultos normais (Maughan et al, 1984; Mayhew et al., 1991; Mayhew et al., 1993).

Entretanto, nosso estudo não apresentou correlação entre as variáveis força e ASTm, e aponta 
urna grande dispersão da amostra. Essa dispersão pode ser influenciada por fatores como o nivel de atividade física de cada sujeito, que influenciaria na coordenação neuromuscular e sobre os mecanismos neurais inibitórios (órgão tendinoso de Golgi). Há também a possibilidade da influencia dos diferentes tipos de fibras musculares predominantes em cada individuo avahado, além do número amostral reduzido.

Segundo Sunnegárdh (1988), a força muscular é influenciada por uma série de variáveis, como :idade, sexo, características antropométricas e, também, pelo grau de atividade física. Neste sentido, mesmo que o critério de seleção amostral tenha sido o sedentarismo durante os últimos 12 meses, não se tem a garantia de que os indivíduos tenham sido expostos aos mesmos estímulos tísicos diários, em suas atividades laborais e de lazer. Cita, também, que os músculos das extremidades, ou dos membros, são constantemente submetidos a esforços de característica dinâmica, o que pode influenciar na sua produção de força, e que a variabilidade na produção de força é especificamente influenciada pelos diferentes tipos de atividade muscular.

De acordo com Sale (1992), a capacidade de exercer força máxima não é determinada somente pelo tamanho dos músculos envolvidos no exercício, mas também pela habilidade do sistema nervoso de ativar apropriadamente estes músculos. Indivíduos não treinados, talvez, não tenham a habilidade de recrutar unidades motoras de alto limiar, o que, em outras palavras, quer dizer que não é possível que tais indivíduos ativem totalmente os músculos agonistas, rerdendo então a relação entre a área muscular e a produção de força máxima.

Segundo Badillo (1997), o órgão tendinoso de Golgi parece limitar a produção de força máxima, através de um mecanismo neural inibitório. Com o treinamento de força, por um processo que não é totalmente conhecido, parece que o limiar dos receptores de Golgi é elevado, permi- tindo maior produção de força e, conseqüentemente, reduzindo sua ação inibitória. Mayhew (1991) afirma que, após um período de treinamento de força, no qual este processo de inibição seria minimizado, a relação entre a ASTm e a produção de força máxima seria mais evidente.

Outro mecanismo que poderia influenciar na produção de força máxima seria a co-contração dos músculos anta-

gonistas ao movimento

avaliado. De acordo com Alguns estudos Sale (1992), esse fenôme- apontam que a no é comum em mo- máxima força vimentos de alta in- voluntária de não treinados para oxtensores do joelho não treinados para o extensores do joelho
movimento específico. eflexores do cotovelo Esta co-contração é são positivamente contra-produtiva, particu- correlacionadas com larmente em trabalhos de a AST $m$ de homens força, devido ao torque adultos normais opositor desenvolvido pelo antagonista, o que diminui o torque máximo na direção do movimento.

Conforme Fleck e Kraemer (1999), as fibras musculares do tipo I (contração lenta) e do tipo II (contração rápida) diferem nos mais diversos pontos, e entre eles está a produção de força por unidade de secção transversa. Ou seja, as fibras musculares do tipo II são capazes de produzir mais força que as fibras do tipo I, quando comparadas na mesma ASTm.

Portanto, os indivíduos testados poderiam apresentar diferenciação entre a predominância dos tipos de fibras musculares, o que poderia ser uma das causas da variabilidade apresentada nos amostra.

Entretanto, há uma série de contradições a respeito deste assunto, em que alguns autores, como Billeter (1992), afirmam que a força produzida não é determinada pelo tipo de fibra muscular predominante, isto é, as fibras musculares do tipo 
I e II apresentam a mesma força relativa. Quando um indivíduo apresenta um percentual maior de fibras musculares do tipo II, ele, conseqüentemente, apresentará maior volume muscular (ASTm). Sendo assim, tal maior volume muscular é que será responsável pelo nível de força mais elevado. Close (1972), mostra em seu estudo que a máxima força por unidade de ASTm é aproximadamente a mesma tanto em músculos com predominância de fibras de contração rápida, como nos músculos com predominância de fibras de contração lenta.

Sendo assim, Maughan (1983) afirma que não se sabe ao certo se as diferenças na produção de força máxima, entre as fibras musculares de contração rápida e lenta, se dão pela maior ASTm das fibras de contração rápida, ou se estas geram maior força máxima relativa por ASTm que as fibras musculares de contração lenta.

Apesar de não ter havido correlação entre as variáveis força máxima dinâmica e estimativa da ASTm, a partir da equação de Gurney (1973), o que impossibilita a modulação da carga a partir desta, sugere-se que outros estudos sejam realizados. Recomenda-se, porém, a utilização de uma amostra mais significativa e homogênea. Pode-se, ainda, utilizar treinamento de força, antecedendo à coleta de dados, como propõe Rutherford (1986), para, assim, diminuir a ação dos mecanismos neurais inibitórios e talvez termos uma relação mais direta entre a força máxima dinâmica e a ASTm.

\section{Referências Bibliográficas}

BADILLO JJG. Fundamientos del Entrenamiento de la Fuerza. Aplicación al alto rendimiento deportivo. Inde Publicaciones, $2^{\text {a }}$ Ed. 1997.

BAECHLE TR. Weight Training: Steps to Success. Human Kinetics, 2 ${ }^{\mathrm{a}}$ Ed. 1998.
BILLETER R, et al. Muscular Basis of Strength. Em: KOMI PV. Strength andpower in sport. Human Kinetics, 1992.

CLOSE RI. Dynamic Properties of Mammalian Skeletal Muscle. Physiol. Review. 52:129 -197, 1972.

CURETON KJ, COLINS MN, HILL DW McELHANNON JM. Muscle hypertrophy in men and women. Med. Sci. Sports Exerc. 20: 338-344, 1988.

FLECK SJ, KRAEMER, W. Fundamentos do Treinamento de Força Muscular. Artes Médicas,

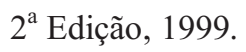

GURNEY JM, JELLIFFE DB. Arm anthropometry in nutritional assessment. Nomogram for rapid calculation of muscle circumference and crosssectional muscle and fat áreas. Am. J. Clin. Nutr. 26:912-915, 1973.

HEYMSFIELD SB, McMANUS C, SMITH J, STEVENS V, NIXON DW. Anthropometric measurement of muscle mass: revised equations for calculating bone-free arm muscle área. Am. J. Clin. Nutr. 36: 680-690, 1982.

KNUTTGEN HG, KRAEMER. Terminology and measurement in exercise performance. J. Appl. Sports Sci. Res. 1: 1 -10, 1987.

LEHMKUHLLD, SMITH LK. Cinesiologia Clínica. Manóle, $4^{\mathrm{a}}$. Edição, 1989.

KRAEMER WJ. Hormonal mechanismis related to the expression of muscular strength and power. In Strength and Power in Sport, ed. PV. Komi, 64-76. Oxford, 1992.

LOHMAN TG, et al. Anthropometric Standardization Reference Manual. Human Kinetics, 1988.

MAUGHAN Rj, et al. Relationships between muscle strength and muscle cross-sectional área in male sprinters and endurance runners. Eur. J. Appl. Physiol. 50:309-318, 1983.

MAUGHAN RJ, et al. Strength and cross-sectional 
área of human skeletal muscle. /. Physiol. 338: 37-49, 1983.

MAUGHAN RJ, et al. The influence of variations fibre composition on muscle strength and crosssectional área in untrained males. /. Physiol. 351:299-311, 1984.

MAYHEW JL, BALL TE, WARD TE, HART CL, ARNOLD, MD. Relationships of structural dimensions to bench press strength in college males./. SportsMed. Phys. Fitness. 31: 135-141, 1991.

MAYHEW JL, PIPER FC, WARE JS. Anthropometric correlates with strength performance among resistance trained athletes. I. Sports Med. Phys. Fitness. 33:159-165,1993.

RASCH PJ, BURKE RK. Cinesiologia e Anatomia Aplicada. Guanabara, 5ª . Edição, 1977.

ROLLAND-CACHERA MF, et al. Body composition assessed on the basis of arm circumference and triceps skinfold thickness: a new Índex validated in children by magnetic resonance imaging. Am.J. Clin. Nutr. 65:17091713, 1997.

RUTHERFORD OM, et al. The Role of Learning and Coordenation in Strength Training. Eur. J. Appl. Physiol. 55: 100 -105, 1986.

SALE DG. Neural adaptation to strength training. Em: KOMI PV. Strength and power in sport. Human Kinetics, 1992.

SUNNEGÂRDH J., ET, et al. Isometric and isokinetic muscle strength, anthropometry and physical activity in 8 and 13 year oíd Swedish children. Eur. J. Appl. Physiol. 58: 291 - 297, 1988

ZATZIORSKY VM. Ciência e Prática de Treinamento de Força. Phorte Editora. I ${ }^{\mathrm{a}}$ Ed. $\mathrm{p}$ 315. São Paulo, 1999.

\section{Notas}

Ronei Silveira Pinto é Mestre em Ciencias do Movimento Humano pela ESEF/UFRGS e Professor da ESEF/UFRGS.

Gianni Rodolfi é Professor de Educação Física formado pela ESEF/UFRGS.

Lucimere Bohn é Graduanda do Curso de Educação Física e Monitora da disciplina de Musculação da ESEF/UFRGS.

Endereço de contato: Laboratorio de Pesquisa do Exercício (LAPEX), ESEF/UFRGS - Rua Felizardo, 750 - Bairro Jardim Botânico - Porto Alegre/RS. 
